\title{
Prevention of Hypothermia of Low Birth Infants Using The Kangaroo Method
}

\author{
Anna Alisjahbana, Ali Usman, Suzi Irawaty, Atte Triyati \\ (Medical Research Unit, Medical School, Padjadjaran University / \\ Hasan Sadikin Hospital, Bandung, Indonesia)
}

\begin{abstract}
Hypothermia is a common problem in neonates and an important contributory factor to neonatal mortality and morbidity. The results of an evaluation involving several health facilities and health personnel showed that thermal control practices were frequently inadequate in the following areas: ensuring a warm environment at the time of delivery, initiation of breastfeeding and contact with mother, bathing, checking the baby's temperature, thermal protection of low birth weight babies, and care during transportation. During kangaroo method (K-M) the infant is in skin to skin contact with the mother. A pilot study conducted on LBW infants discharged from the General Hospital in Bandung, and follow-up for 4 consecutive weeks showed the skin temperature to be well maintained. Weight increase was more stable compared to the control group and there were no re-hospitalization within the observation period. $\mathrm{K}-\mathrm{M}$ has a simple and natural appeal, cold stress can be avoided; perhaps the most important benefit of $\mathrm{K}-\mathrm{M}$ is improvement of lactation. In developing countries especially rural areas, temperature regulation and maintenance of lactation using K-M may be lifesaving for very low birth infants. [Paediatr Indones 1998; 38:205-214]
\end{abstract}

\section{Introduction}

The 45th World Health Assembly on Child Health And Health of The Newborn inn 1992 recognized that significant improvements in health of the newborn could be achieved by integrating safe motherhood initiatives with appropriate care of newborn, namely improving maternal nutrition, controlling perinatal infections, adapting resuscitation and thermal control principles to local circumstances and ensuring that breastfeeding starts immediately after birth.

The kangaroo method (K-M) was first introduced by Rey and Martinez in Colombia, South America, and further studies were conducted by Anderson in 1986) and 
Colonna in 1990. Mozambique doctors with staff of the WHO collaborating Center for Maternal and Child Health, Trieste, Italy for further implementation, evaluation and improving the K-M. The K-M consists of placing the low birth weight (LBW) infants between the mothers breasts in a prone or vertical position (see Figures 1 and 2) rather than in an incubator. Both expert groups were confident that the $\mathrm{K}-\mathrm{M}$ was an appropriate strategy to improve immediate as well as long-term survival and well being of LBW infants especially those with a birthweight of less than 1500 grams. The Italian expert group divides the $\mathrm{K}-\mathrm{M}$ into two groups e.g.; the $\mathrm{K}-\mathrm{M}$ as an alternative method with respect to the conventional system, and the second alternative is the K-M method as a complementary method to the conventional system. ${ }^{1}$ Although no disadvantages of K-M for LBW infants have ever been published, there are still some questions that need to be addressed such as: how the K-M method is perceived by users and how can cultural resistance and differences be overcome? This study aimed to collect information on home care of low birth weight babies using the K-M with special attention to the feasibility and acceptability of the method by mothers.

\section{Methods}

This report is the result of a pilot study conducted at the Hasan Sadikin Hospital, Bandung. The study started in January 1996, the output of the study was to collect information on home care of low birth weight babies using the K-M with special attention to the feasibility and acceptability of the method by LBW infant mothers. This information will be used to develop technical guidelines and information material for mothers and community midwives.

Mothers delivered LBW infants who were discharged from Hasan Sadikin Hospital were randomly assigned into the control group (without $\mathrm{K}-\mathrm{M}, 50$ mothers and their infants) and the intervention group (with K-M, 50 mothers with their infants). The two groups had the same inclusion criteria and research method except for the $\mathrm{K}-\mathrm{M}$ applied. They were followed up until 4 weeks.

\section{Results}

The characteristics of infants included in the study are depicted in Table 1. It shows the difference was significant only for gestational age. Subjects included in the intervention group had lower gestational age compared to the control group although both groups had a mean birth weight of less than 2500 grams. The control group had more cases of small for gestational age, and the mean birth weight was slightly higher although not significantly different than the treated group. 

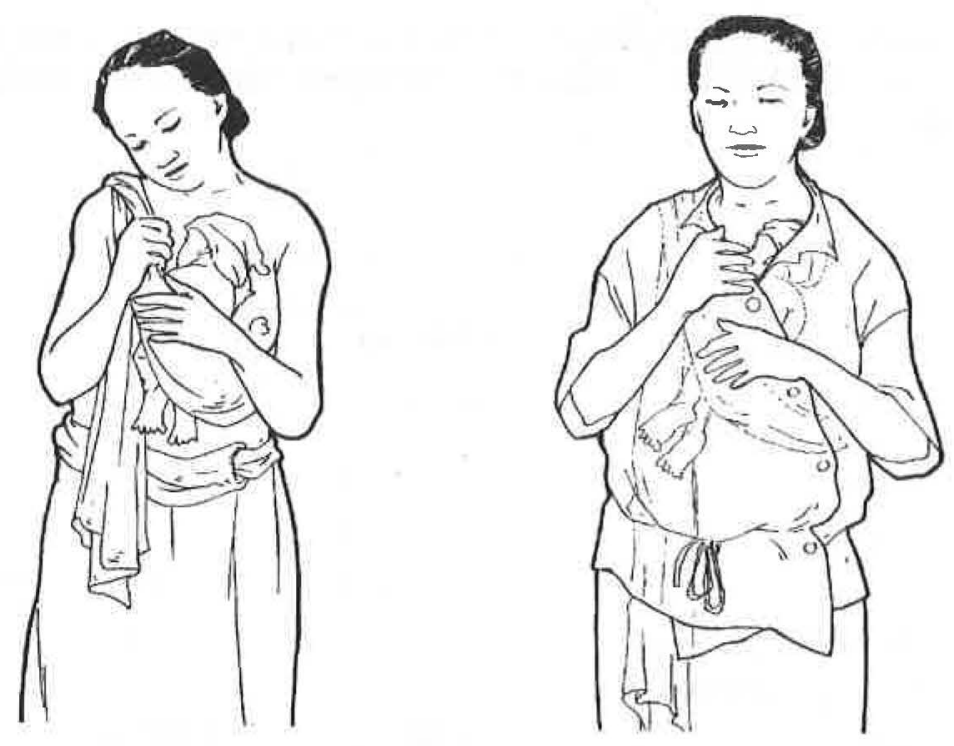

Figure 1. Caring for a low birth weight infants using the Kangaroo Method
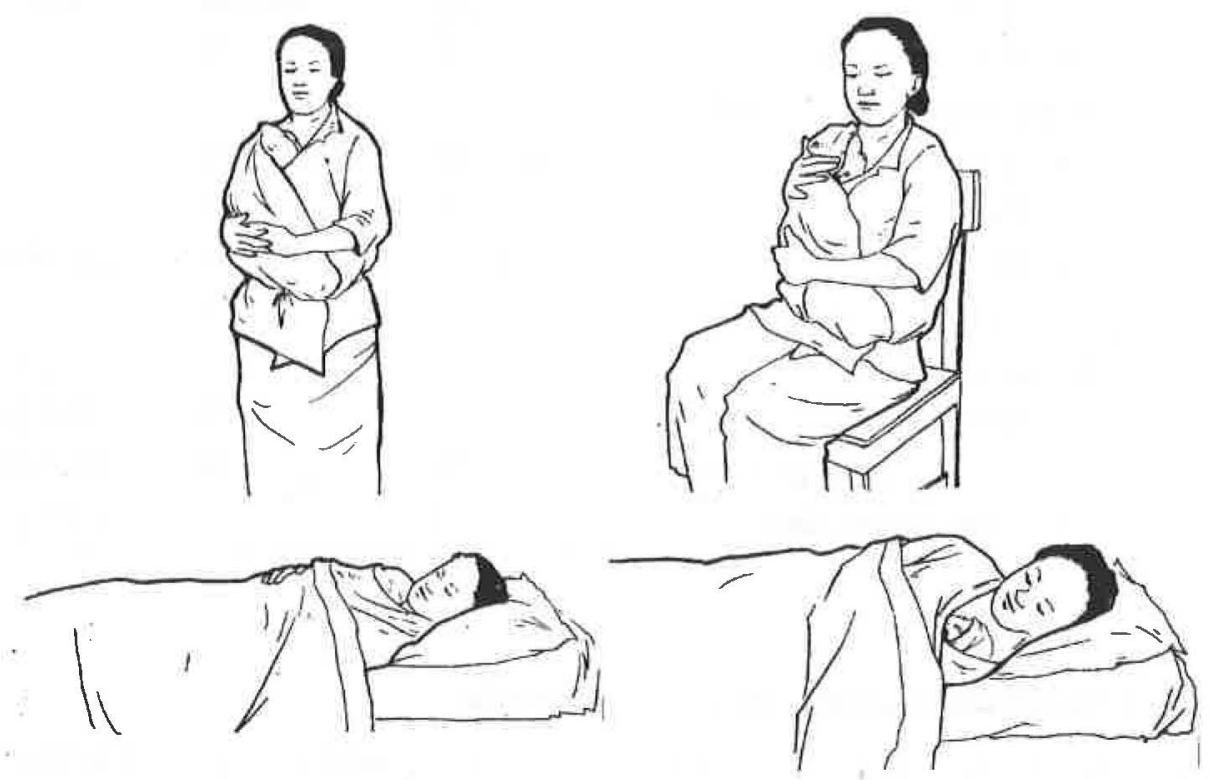

Figure 2. Kangaroo Method in practice; standing position, sitting position and sleeping. 
The mean weight on hospital discharge was almost the same as mean birthweight. It shows that most infants had regained their birthweight already at the start of the intervention study.

Table 1. Characteristics of low birth weight infants by group

\begin{tabular}{cccc}
\hline Variables & K-M group \\
& $N=50$ & $\begin{array}{c}\text { Control group } \\
N=50\end{array}$ & $P$. value \\
\hline
\end{tabular}

1. Gestational age (weeks)

- Range

- Mean

- SD

- >37 week (n)

2. Low birth weight (grams)

- Range

- Mean

- SD

- >2000 gram (n)

3. Weight on discharge (grams)

- Range

- Mean

- SD

- >2000 gram (n)
28-40

34.13

3.14

11

$\begin{array}{rrr}1050-2260 & 1200-2500 & \\ 1829.60 & 1928.20 & \\ 312.46 & 281.44 & 0.16287 \\ 20 & 27 & \end{array}$

1055-2230

1833.06

242.03

17

$1300-2200$

1909.38

213.58

0.12726

29

4. Intrauterine growth

- Small for date

$\begin{array}{rrr}11 & 25 & 0.00370 \\ 35 & 23 & 0.0155 \\ 4 & 2 & 0.6777\end{array}$

\section{Body temperature recorded during home visits}

Body temperature was recorded daily by the mother and weekly by the interviewer.

Figure 3 shows the result of the interviewer recording during home visits, the control 
group was compared with the intervention group. Body temperature was relatively stable after the fourth week. This was not the case for the control group, here about $4 \%$ still had hypothermia and $18 \%$ showed signs of cold stress.
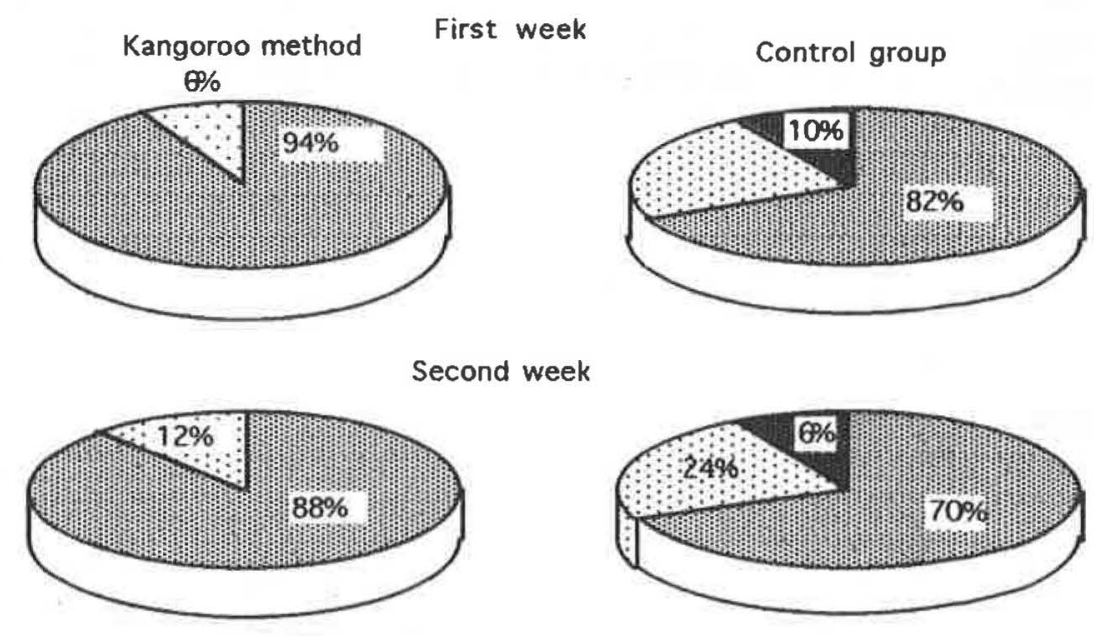

Third week
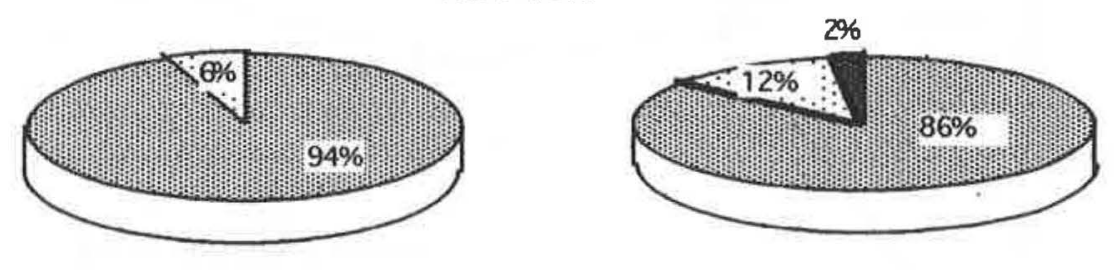

Forth week
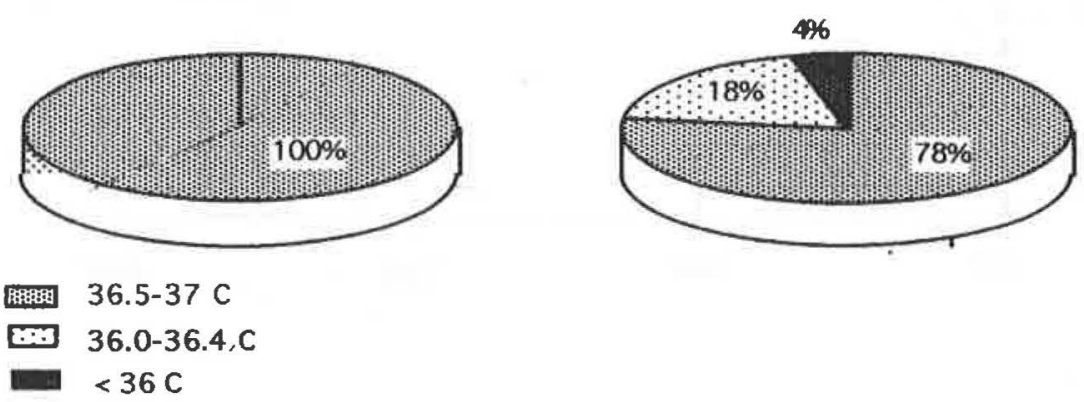

Figure 3. Temperature of newborns with Kangaroo Method and controls. 


\section{Weight increase}

Weight increase was measured regularly once a week for a period of 4 weeks. Research interviewers visited the house, measured weight, and collected other information of the infants condition. The results show a more stable weight increase in the intervention group, this was not shown in the control group (Figure 4). It need to be mentioned here that all infants received exclusive breast feeding.

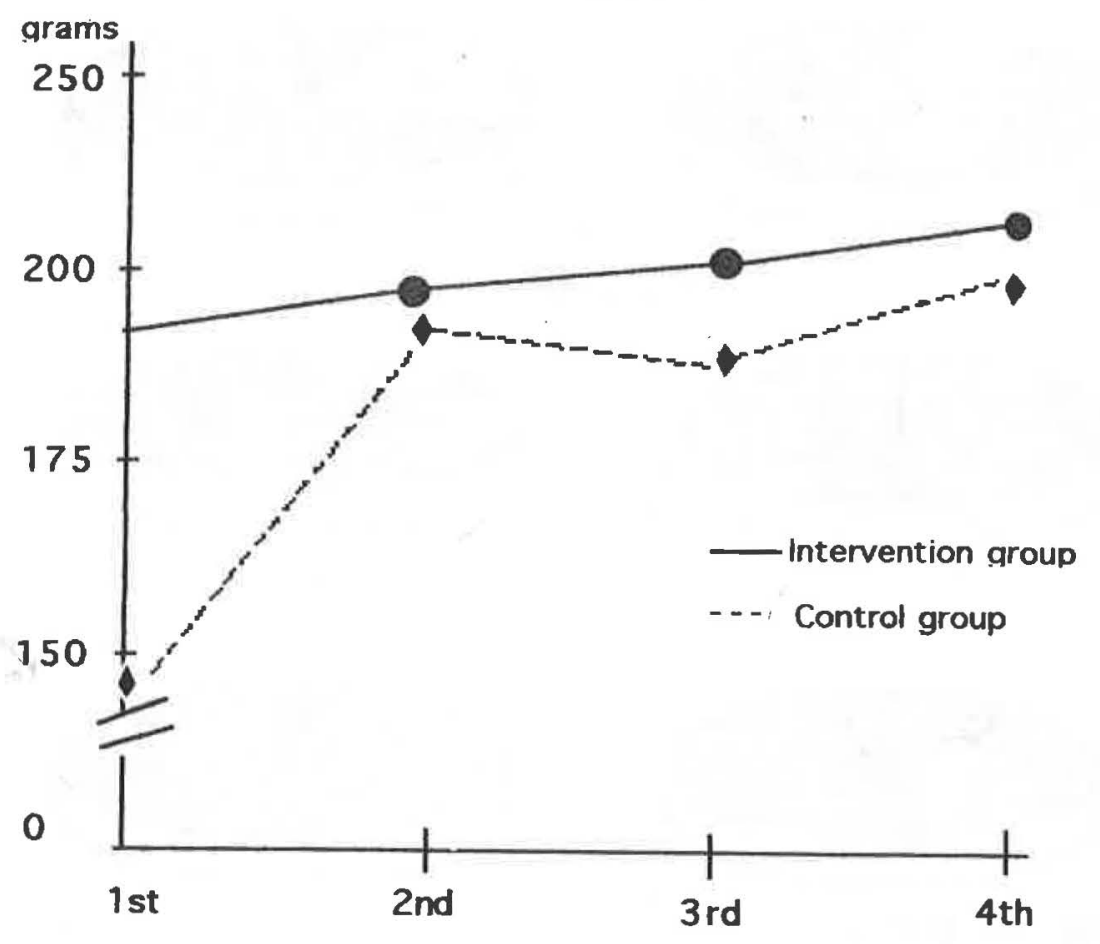

Figure 4. Body weight increase during 4 consecutive weeks in intervention and control groups. 


\section{Mothers perceptions and attitude}

Although the research team advice the mother to provide K-M for at least 12 hours a day, it was interesting to note that mothers decided to applied the $\mathrm{K}-\mathrm{M}$ according to their convenience. The result was that the number of hours using the $K-M$ among mothers differed. Based on the number of hours the mother was providing the $\mathrm{K}-\mathrm{M}$, the intervention group was divided into 4 subgroups, i.e. Group I ( $\mathrm{K}-\mathrm{M}<5$ hours/day); Group II (K-M 5-<10 hours/day); Group III (K-M 10-<15 hours/day); and Group IV (K$M>15$ hours/day). The effect on increase in weight can be seen in Table 2.

Table 2. Distribution of gestational age, birth weight, and body weight by duration of K-M

\begin{tabular}{lcccccccc}
\hline $\begin{array}{l}\text { Group } \\
\begin{array}{c}\text { duration of } \\
\text { K-M/day) }\end{array}\end{array}$ & $\mathrm{n}$ & Gestation & $\mathrm{BW}(\mathrm{g})$ & Wd $(\mathrm{g})$ & \multicolumn{4}{c}{ Mean body weight $(\mathrm{g})$} \\
\cline { 2 - 8 } & $\begin{array}{c}\text { Mean } \\
(\mathrm{SD})\end{array}$ & $\begin{array}{c}\text { Mean } \\
(\mathrm{SD})\end{array}$ & $\begin{array}{c}\text { Mean } \\
(\mathrm{SD})\end{array}$ & $\begin{array}{c}\text { Week 1 } \\
\text { Mean } \\
(\mathrm{SD})\end{array}$ & $\begin{array}{c}\text { Week 2 } \\
\text { Mean } \\
(\mathrm{SD})\end{array}$ & $\begin{array}{c}\text { Week 3 } \\
\text { Mean } \\
(\mathrm{SD})\end{array}$ & $\begin{array}{c}\text { Week } 4 \\
\text { Mean }\end{array}$ \\
\hline I(<5 hrs) & 13 & 33 & 1840 & 1859 & 2073 & 2276 & 2527 & 2794 \\
II (5-9 hrs) & 8 & 35 & 1741 & 1826 & 1931 & 2107 & 2275 & 2400 \\
& & $(1.6)$ & $(264)$ & $(159)$ & $(187)$ & $(246)$ & $(260)$ & $(31)$ \\
III (10-14 hrs) & 8 & 34 & 1720 & 1678 & 1893 & 2093 & 2368 & 2481 \\
& & $(2.5)$ & $(182)$ & $(163)$ & $(163)$ & $(290)$ & $(140)$ & $(463)$ \\
IV $(\geq 15 \mathrm{hrs})$ & 1 & 36 & 1620 & - & 1700 & 1900 & 2200 & 2550 \\
\hline
\end{tabular}

$\mathrm{BW}=$ birthweight; $\mathrm{Wd}=$ Weight at discharge

Table 3. Distribution of pulse, respiration, and temperature at the first home visit by group

\begin{tabular}{llccc}
\hline $\begin{array}{c}\text { Group } \\
\text { (duration of } \\
\text { K-M/day) }\end{array}$ & $\mathrm{N}$ & $\begin{array}{c}\text { Pulse rate } \\
\text { Mean (Range) }\end{array}$ & $\begin{array}{c}\text { Respiratory rate } \\
\text { Mean (Range) }\end{array}$ & $\begin{array}{c}\text { Temp. }\left({ }^{\circ} \mathrm{C}\right) \\
\text { Mean (Range) }\end{array}$ \\
\hline I (<5 hrs) & 13 & $146(140-160)$ & $44.6(40-52)$ & $36.6(36.2-37.2)$ \\
II (5-<10 hrs) & 8 & $140(130-150)$ & $44(38-48)$ & $36.7(36.0-37.0)$ \\
III (10-15 hrs) & 8 & $142(120-150)$ & $46(40-62)$ & $36.9(36.6-37.3)$ \\
IV (>15 hr) & 1 & 140 & 52 & 37.2 \\
\hline
\end{tabular}


From Tables 2 and 3 , it can be seen that no difference in vital signs and weight increase within each subgroup was found. However the numbers were to small for statistical analysis. A qualitative survey using focus group discussion was conducted at the end of the observation period to study mothers perception and acceptance of the method. The result showed that 34\% of mothers felt happy, $42 \%$ mothers were not very sure about her feelings and $24 \%$ were afraid that they could not provide baby's needs. Mothers who were in the $<5$ hours subgroup were reluctant to apply the K-M because they felt that their activities became limited. Most of the mothers who still continued using the K-M complained that they also found it not very practical. However they all agreed that the K-M benefited the baby especially after they found out that the baby's weight was increasing and the baby looked healthy. During the observation period no referrals due to babies condition were made.

\section{Discussion}

Hypothermia, defined as body temperature below $36.5^{\circ} \mathrm{C}$, is a frequent finding in newborn babies and particularly in LBW babies in both developed and developing countries. $^{2}$ An evaluation of knowledge and practices of health professionals on the thermal control of newborns was carried in 7 countries: Brazil, India, Indonesia, Kazakstan, Mozambique, Nepal and Zimbabwe. The evaluation was conceived from a one-day training course on thermal control involved 28 health facilities and 260 health professionals (61 doctors, 199 nurses and midwives). The results showed that thermal control practices were frequently inadequate in the following areas: ensuring a warm environment at the time of delivery, initiation of breast feeding and close contact with mother. Knowledge on thermal control was also insufficient, especially concerning the physiology of thermo-regulation and criteria for defining hypothermia. This findings were consistent across the countries. ${ }^{3}$ From the evaluation sheet for health facilities, it also shows that low reading thermometer was not available. Not all centers reported breast feeding immediately after birth and the "Kangaroo Method" was not practiced. Bathing the baby immediately after birth is still a common practice. In the delivery room not all hospitals were providing radiant warmers for the newborn infant. In one hospital the delivery room was using air condition. The general response of the participants concerning the training was good and satisfactory but the one-day training was felt too short.

In many developing countries exclusive breast feeding and thermal control especially in rural areas due to lack of electricity and formula feeding using appropriate technology is very important. ${ }^{4,5}$ Temperature regulation and maintenance of lactation by K-M may be lifesaving for preterm infants. From several studies in industrial countries it was found that the lactation period is also significantly longer in mothers using the K-M. At 6 months of age infants who had skin contact cried significantly less than 
the control group. ${ }^{4}$ For developed countries K-M care is certainly not an alter-native to intensive care for life-threatening illness but offers a means of helping mothers and fathers to overcome the feeling of separation and inadequacy that frequently accompany preterm delivery. ${ }^{5}$

In Indonesia more than $60 \%$ of women deliver at home by midwives and traditional birth attendant (TBA) while the incidence of LBW is $14 \%$. Hypothermia in very low birth weight (VLBW) infants is common. Neonatal mortality is high and causes of early neonatal death include hypothermia and infection. An intervention study "The risk approach as a primary health care strategy" conducted in Tanjungsari in 1988-1990 revealed that all infants < 1500 grams died because of hypothermia and/or infection. ${ }^{6}$ To anticipate negative outcome, the study recommended to refer all newborns with a birth weight $<2000$ grams. There is no doubt that the need to study appropriate and effective care for LBW infants is greater where the incidence of LBW infants is high and the risk for negative events affecting survival or subsequent quality of life is higher such as in Indonesia.

Indonesia is country with a variety of ethnic groups, cultural habits and behaviors; it is not surprising that child care differs between ethnic groups. Putting the baby directly through skin to skin contact is not a habit in many parts of Indonesia. In some areas (Irian Jaya), women use to carry their young infant on the back instead at her breast - to have her hands free to work on the land. During a pilot survey, women in West Java find it uneasy to have a naked baby immediately at her breast.

The mother's body is an efficient and zero-cost heat source and provide a constant microclimate free from the risk of hyperthermia due to overheating in incubators (in case of malfunction). The K-M encourage early contact between mother and newborn and encourages early breastfeeding. The close skin contact between mother and infant may improve the overall psychomotor performance of the baby in response to sensoric stimuli received from the mother. The K-M has also shown positive effects in terms of workload reduction for neonatal ward staff and consequently a greater attention can be paid for more infants.

The results of the pilot study at Hasan Sadikin Hospital showed that low birth weight infants increased in body weight faster compared to infants cared using conventional methods. Body temperature also became more stable to normal levels within a week. The study did not found any baby who were re-hospitalized due to septicemia. From this result we conclude that the K-M can be recommended to be applied in field condition. An operational study will further be developed to applied the K-M in other provinces e.g. South Sumatra, North Sumatra, and South Sulawesi. The Kangaroo Method is also integrated in essential neonatal care and the mother and baby package program conducted in some provinces in Indonesia funded by the World Bank and the Department of Health. 


\section{Acknowledgment}

The study is sponsored by the World Bank via the Department of Health (Population V, 1991-1996)

\section{References}

1. Davanzo R. Care of the low birth weight infants with the Kangaroo mother method in developing countries. Bureau of International Cooperation in Maternal and Child Health. WHO Collaboration Center for Maternal and Child Health. Trieste, Italy, 1993.

2. Thermal control of the newborn: a practical guide. WHO/FHE/MSM/93.2.

3. Dragovich D, Tamburini G, Alisjahbana A, et al. Thermal control of the newborn: knowledge and practice of health professionals in seven countries. Acta Paediatr 1997;86: 645-50.

4. Whitelaw, Heisterkamp, Leath K Acolet D, Richards M. Skin contact for very low birthweight infants and their mothers. Arch Dis Childh 1988; 63:1377-81.

5. Whitelaw. Kangaroo baby care, just a nice experience or an important advance for preterm infants. Pediatrics 1990;85: 604-5.

6. Alisjahbana A. The risk approach as a primary health care strategy by traditional birth attendant in Tanjungsari. Rotterdam: Erasmus University, 1993. 